\title{
Patients' understanding of how genotype variation affects benefits of tamoxifen therapy for breast cancer
}

\author{
Noel T. Brewer ${ }^{1,2}$, Jessica T. DeFrank ${ }^{1}$, Wing K. Chiu ${ }^{2}$, Joseph G. Ibrahim ${ }^{2}$, Christine M. \\ Walko $^{2,3,4}$, Peter Rubin ${ }^{5}$, Oludamilola A. Olajide ${ }^{6}$, Susan G. Moore ${ }^{6}$, Rachael E. Raab ${ }^{7}$, \\ Daniel R. Carrizosa ${ }^{8}$, Steven W. Corso ${ }^{9}$, Garry Schwartz ${ }^{10}$, Jeffrey M. Peppercorn ${ }^{11}$, \\ Howard L. McLeod ${ }^{2,3,4}$, Lisa A. Carey², and William J. Irvin Jr. ${ }^{2,3,12}$ \\ ${ }^{1}$ Department of Health Behavior, Gillings School of Global Public Health, University of North \\ Carolina (UNC) \\ ²Lineberger Comprehensive Cancer Center, UNC \\ ${ }^{3}$ Institute for Pharmacogenomics and Individualized Therapy, UNC \\ ${ }^{4}$ Eshelman School of Pharmacy, UNC \\ ${ }^{5}$ Conehealth Cancer Center \\ ${ }^{6}$ Rex Hematology/Oncology Associates \\ ${ }^{7}$ East Carolina University \\ ${ }^{8}$ Carolinas Medical Center \\ ${ }^{9}$ Palmetto Hematology/Oncology \\ ${ }^{10}$ Northeast Oncology Associates \\ ${ }^{11}$ Duke Cancer Institute \\ ${ }^{12}$ Bon Secours Cancer Institute
}

\section{Abstract}

Background-CYP2D6 is a critical enzyme in the metabolism of tamoxifen and potentially a key determinant in breast cancer outcomes. Our study examined patients' beliefs about how CYP2D6 genotype would affect their prognoses.

Methods-Women enrolled in a pharmacogenomic clinical trial and on tamoxifen for prevention or treatment of breast cancer underwent CYP2D6 genotyping (EM=extensive, IM=intermediate, $\mathrm{PM}=$ poor metabolizing alleles). The informed consent said that the purpose of the trial was to examine effects of dose adjustment based on genotype, but that clinical benefits were uncertain. Our embedded sub-study surveyed 320 patients prior to receiving their genotypes. We

For correspondence with journal: Jessica DeFrank, University of North Carolina, 325 Rosenau Hall CB 7440, Chapel Hill, NC 27516, jessica_defrank@unc.edu, (t) 919-843-4726; Corresponding author once published: Noel T Brewer, PhD, University of North Carolina, 325 Rosenau Hall CB 7440, Chapel Hill, NC 27516, ntb@unc.edu.

Potential Conflicts of Interest: Dr Rubin receives remuneration from Eisai Novartis. Dr Schwartz has stock ownership in Pharmacyclics, Seattle Genetics, Pfizer, and Allergan. Drs. Brewer, DeFrank, Chiu, Ibrahim, Walko, Olajide, Moore, Raab, Carrizosa, Corso, Peppercorn, McLeod, Carey and Irvin have no disclosures. 
experimentally manipulated 6 vignettes to describe hypothetical tamoxifen treatment (no or yes) and hypothetical genotype (EM, IM or PM). For each vignette, women gave their perceived recurrence risk (RR; 0-100\%).

Results-Women believed that genotype would not affect their RR if they did not take tamoxifen ( $p=.06$ ). However, women believed that if prescribed tamoxifen, genotype would affect their RR (22\% if EM, 30\% if IM and 40\% if PM, $p<.001$ ).

Conclusion-Women believed that extensive tamoxifen metabolizers had better prognoses, despite study materials stating uncertainty about any benefit. The rapidly changing nature of genomic science calls for caution when communicating clinical utility.

\section{Keywords}

Pharmacogenomics; risk communication; breast cancer; genomics; tamoxifen

\section{Introduction}

New genomic markers will increasingly inform clinical care, but some may become available to patients prior to clinical validation. Cytochrome P450 2D6 (CYP2D6) is a critical enzyme in the metabolism of tamoxifen [1-3], however studies are conflicting regarding CYP2D6 as a tamoxifen efficacy biomarker. For this reason, CYP2D6 genotyping currently does not meet evidence for clinical use [4]. However, in 2009, when data were just emerging on this subject, a national community-based survey suggested that one-third of oncologists had previously ordered CYP2D6 genotyping for use in clinical decision making [5].

While pharmacogenomics is an emerging area for breast cancer research, little is known regarding how well patients understand pharmacogenomics or the rationale for clinical trial research in this area [6]. Our pilot study suggested that after informed consent, a substantial number of participants reported a strong degree of understanding of pharmacogenomics research, but remained confused about several aspects of the study [7]. In other areas of oncology research, trial participants have often expressed high expectations of direct personal benefit of the intervention, regardless of scientific uncertainty $[8,9]$.

Previous work has shown that, in some cases, women resist or even ignore breast cancer risk information [10], while other studies show highly accurate responses to risk information [11]. Thus, it is not clear whether patients would understand the risk information provided by this new genomic test. Our previous research has shown that women's understanding of recurrence risk as provided by genomic test results is strongly associated with both hypothetical and actual treatment choices [11,12]. These findings highlight the importance of studying the patient's perspective of receiving and understanding test results based on genomic information. Assessing patients' reactions to complex genomic risk information may guide the development of communication strategies to support patient understanding and adherence to treatment.

Our experimental study sought to examine patients' beliefs about how hypothetical genotype information would affect their perceived recurrence risk. Given the known benefits of 
tamoxifen, we hypothesized that receipt of this treatment (hypothetical) would be associated with a reduction in perceived recurrence risk. Furthermore, we hypothesized that patients would expect a reduction in recurrence risk only in the setting of tamoxifen treatment; we would not expect to find this relationship in the setting of no tamoxifen treatment.

\section{Materials and Methods}

\section{Participants}

In Lineberger Comprehensive Cancer Center trial 0801, women on tamoxifen for prevention or treatment of breast cancer at a university hospital and several community clinics underwent CYP2D6 genotyping. We have previously reported the methods for the pilot study [13]. Patients with any intermediate or poor metabolizing (IM or PM) alleles received increased tamoxifen doses of 40mg daily, but patients homozygous for extensive metabolizing (EM) alleles received routine doses of $20 \mathrm{mg}$ daily. The informed consent document stated that the purpose of the study was to see if dose adjustment could raise endoxifen concentrations in reduced metabolism patients, but that it was not clear whether dose escalation would provide clinical benefit. In this embedded sub-study performed between April 2009 and September 2010, patients completed a survey prior to receiving their genotype.

Participants were women who had been taking tamoxifen for at least 4 months (but no more than 4.5 years) to prevent or treat breast cancer (ductal carcinoma in-situ or invasive). The trial included women with Eastern Cooperative Oncology Group (ECOG) performance status of 0 to 2 and an expected survival of at least 6 months. Exclusion criteria were being on any of the following CYP2D6 inhibiting medications: amiodarone, haloperidol, indinavir, ritonavir, quinidine, duloxetine, paroxetine, bupropion, or fluoxetine. We collected these data directly from patients and their treating physicians through medication review by the nurse study coordinator.

Of 377 eligible patients, 320 patients completed the survey (85\%), and 57 returned incomplete surveys. Of the incomplete surveys, 32 had no data for the section of the survey germane to this study, and 25 had partial data. Patients with incomplete survey data were similar on all variables we examined compared to patients who completed the survey. Most respondents who completed the survey were white $(81 \%)$ and were on tamoxifen for treatment of invasive breast cancer (85\%) (Table 1); mean time on tamoxifen treatment was 16 months. Fewer patients (14\%) had a DCIS diagnosis and were on tamoxifen for prevention of recurrence. About one-third (32\%) were premenopausal. Patients' actual genotypes were 35\% for EM, 58\% for IM and 5\% for PM.

\section{Procedures}

The University of North Carolina institutional review board reviewed and approved the study. The informed consent document that all patients signed stated: "The purpose of this research study is to learn if the increased dose of tamoxifen in intermediate and poor metabolizers will have an effect on the patient's endoxifen level. It is possible you will receive no benefit from participating in this study. Your participation will help us answer the 
question of whether we can raise the level of the active metabolite of tamoxifen in women who otherwise do not break-down the drug as well, but it is not clear that this will actually provide any benefit."

We used a within-subjects experimental design to assess patients' beliefs about how CYP2D6 genotype and tamoxifen treatment would affect patients' perceptions of breast cancer recurrence risk. We asked them to evaluate 6 hypothetical vignettes prior to knowing their genotype. We experimentally manipulated the vignettes to describe whether women hypothetically received tamoxifen treatment (no or yes) and their hypothetical genotype (EM, IM, PM). Use of hypothetical vignettes is a well-accepted methodology for understanding response to new cancer testing [12, 14-15]. Vignettes used in our study appear in the appendix.

\section{Measures}

The following instructions appeared at the top of the survey vignettes (Appendix A): "A new genotype test can say how well your body uses (metabolizes) tamoxifen. Extensive metabolizers (EM) may get the full benefit from tamoxifen. Intermediate metabolizers (IM) may get some benefit from tamoxifen. Poor metabolizers (PM) may get the least benefit from tamoxifen." For each combination of genotype and tamoxifen treatment (independent variables), participants indicated what they thought their chance of cancer recurrence would be, from $0 \%$ to $100 \%$ (dependent variable). For example, the poor metabolizer/no tamoxifen vignette read, "If you don't take tamoxifen and your genotype test shows you are a poor metabolizer, what do you think would be your chance of recurrence?" The instructions asked participants to give their best answer if they were unsure.

\section{Data Analyses}

We analyzed the data using a 3 (genotype) $\times 2$ (receipt of tamoxifen) repeated measures ANOVA, using two-tailed tests with a critical alpha of .05. Data met the assumptions for repeated measures ANOVA, normality (Shapiro-Wilk, W=0.93) and homogeneity of variance (Levene's test, $p=0.13$ ). We probed the interaction by looking at ANOVAs for effect of genotype, for the two hypothetical tamoxifen treatment groups (received or did not receive), using two-tailed Bonferroni-adjusted tests. We conducted sensitivity analyses stratifying by actual genotype and type of breast disease (DCIS or invasive). Effect sizes are reported partial eta-squared $\left(\eta^{2}\right)$ which can be interpreted as proportion of variance explained. As data were completely within-subjects, controlling for participant characteristics was unnecessary. Statistical analyses were performed with SAS version 9.3 statistical software (SAS Institute Inc., Cary, NC).

\section{Results}

Overall, patients expected tamoxifen to lower their breast cancer recurrence risk (RR) from $49 \%$ to $31 \%\left(\mathrm{~F}_{1,638}=92, p<.001\right)$ (Figure 1). Patients expected that being poorer metabolizers, as indicated by genotype status, would increase recurrence risk $\left(F_{2,638}=69\right.$, $p<.001$ ). Risk perceptions were more sensitive to genotype if tamoxifen were prescribed than if not (interaction, $\mathrm{F}_{2,638}=20, p<.001$ ). 
For the setting of no tamoxifen treatment, patients believed that merely being able to metabolize tamoxifen would provide no benefit in reducing their RR (shallow slope on the left panel of Figure 1, partial $\eta^{2}=0.00$ ). For the no-tamoxifen/EM condition, women estimated their RR to be $47 \%$ on average, $48 \%$ in the no-tamoxifen/IM condition, and 53\% in the no-tamoxifen/PM condition. These three RRs were not statistically significantly different from one another $(p=0.06)$. Effects sizes for comparisons in the no-tamoxifen treatment setting were partial $\eta^{2}=.05$ for EM vs IM; partial $\eta^{2}=.15$ for IM vs PM; and partial $\eta^{2}=.20$ for PM vs EM.)

For the setting of tamoxifen treatment, patients believed that genotype would have an effect on their RR (steep slope on right half of Figure 1, partial $\eta^{2}=.08$ ). For the tamoxifen/EM condition, women estimated their RR to be $22 \%$ on average, $30 \%$ in the tamoxifen/IM condition, and $40 \%$ in the tamoxifen/PM condition $(p<.001)$. Each of these conditions differed from one another $(p<.001)$. Effects sizes for comparisons in the tamoxifen treatment setting were partial $\eta^{2}=.34$ for EM vs IM; partial $\eta^{2}=.40$ for IM vs. PM; and partial $\eta^{2}=$. 71 for PM vs. EM.) Our sensitivity analyses showed the same pattern of findings (effect of hypothetical genotype was present only in the setting of tamoxifen treatment) when stratifying by patients' breast disease type (DCIS vs. invasive) and their actual genotype. Null findings for the PM genotype subgroup may have been due to small cell sizes ( $\mathrm{n}=16)$.

We also examined consistency in perceived RR for the three conditions with hypothetical tamoxifen treatment. Most patients $(n=228,71 \%)$ responded that EM would have a lower RR than IM. Some said that RR for EM and IM would be equal $(n=65,20 \%)$, but few said that EM would have a higher RR than IM ( $n=27,9 \%)$. Similarly, most patients $(n=234$, $73 \%$ ) responded that IM would have a lower RR than PM. Some said that RR for IM and PM would be equal $(n=59,19 \%)$, but again few said that IM would have a higher RR than $\operatorname{PM}(n=27,8 \%)$.

\section{Discussion}

Our data suggest that participants in pharmacogenomic trials may have a high expectation of clinical benefit despite emphasis in informed consent documents on the scientific purpose of the research and the uncertainty of any direct benefit. Women participating in our study understood that tamoxifen reduced breast cancer risk. They also understood that, in the absence of tamoxifen treatment, genotype alone had no effect on recurrence risk. However, despite consent materials that described uncertainty about CYP2D6 benefit in clinical decision making, the participants believed that this benefit was likely to be larger for extensive metabolizers in the setting of tamoxifen treatment. Whether or not this benefit is indeed larger for extensive metabolizers is an active area of scientific exploration and controversy.

Our findings are consistent with previous research suggesting that patients are highly receptive to genomic risk information $[11,13,16]$. Our previous study showed that women placed more emphasis on genomic test results for predicting cancer recurrence risk compared to standard pathological markers (e.g. cancer stage) when information from these two sources conflicted [13]. These findings differ markedly from previous work on 
communicating with women about $B R C A 1 / 2$ mutations that found that women resisted information from these tests, overestimating their actual risk [10]. It may be that women think differently about information relevant to treatment for a current disease (e.g., CYP2D6) than they do about risk for future disease (e.g., BRCA 1/2).

Recent research has questioned whether high expectations of direct benefit, which patients consistently report in oncology trials, are due to a "therapeutic misestimation" among participants (suggesting that patients misunderstand the purpose of the trial, undermining the validity of the informed consent process) or are expressions of optimism for the best possible outcome [17-19]. Our study was not designed to test this complex question, but highlights the potential for patients to misunderstand what new genomic tests can offer [20,21]. This misunderstanding could bias patients to assume that genomic information is valid, without understanding what is needed for tests to move into routine clinical care. Also, the potential misunderstandings identified here may lead patients to request the test from physicians and affect their willingness to take or continue tamoxifen in the absence of clinical evidence to guide practice at this time.

\section{Strengths and Limitations}

Study strengths are the inclusion of patients who were part of an active clinical trial to adjust tamoxifen dosing and a large clinical sample. Limitations include the use of hypothetical vignettes, though we know of no other way to reasonably assess women's understanding of the potential risk reduction associated with CYP2D6 genotype. It is unclear to what extent that language in the survey instructions led patients to believe tamoxifen worked best (or only) for extensive tamoxifen metabolizers (the vignette instructions reminded participants to the purpose of the trial -- that poor metabolizers may get the least benefit from tamoxifen treatment whereas extensive metabolizers may get the most benefit). Patients may have responded to the vignettes differently had the survey not restated the potential benefit of tamoxifen metabolizer status. Another interpretation is that responses to the vignettes indicated that patients found the hypothesis tested in the trial to be credible. It is also plausible that communications between the physicians and their patients about the purpose of the trial could have influenced patients' beliefs. Lastly, patients from the clinics we studied may be less diverse than patients from other clinical settings.

\section{Clinical Implications}

Patients are highly receptive to receiving genomic risk information. Our study adds to an existing literature examining the lay population's beliefs about the clinical utility of new genomic technology. Findings reinforce the importance of translating and communicating the purpose of genomic trials to patients. Whether CYP2D6 testing will be clinically useful in planning tamoxifen treatment for patients is a topic of active debate. While the oncology community continues to explore this question, caution should be used when communicating clinical utility and results of novel genomic assays to patients.

\section{Acknowledgments}


at Chapel Hill Investments for the Future Grant No. 6231, Laboratory Corporation of America, Roche Diagnostics, American Cancer Society.

\section{Appendix A. Study vignettes}

A new genotype test can say how well your body uses (metabolizes) tamoxifen.

- Extensive metabolizers may get the full benefit from tamoxifen.

- Intermediate metabolizers may get some benefit from tamoxifen.

- $\quad$ Poor metabolizers may get the least benefit from tamoxifen.

For each combination, please say what you think your chance of cancer recurrence would be (from $0 \%$ to $100 \%$, with $0 \%$ meaning your cancer will never come back). If you are unsure, give your best answer.

\begin{tabular}{cll}
\hline \multicolumn{1}{c}{ If you } & and your genotype test shows you are a & $\begin{array}{l}\text { What do you think would be your chance of } \\
\text { recurrence? }\end{array}$ \\
\hline 1. don't take tamoxifen & extensive metabolizer & \% chance \\
\hline 2. don't take tamoxifen & intermediate metabolizer & $\%$ chance \\
\hline 3. don't take tamoxifen & poor metabolizer & $\%$ chance \\
\hline 4. take tamoxifen & extensive metabolizer & \% chance \\
\hline 5. take tamoxifen & intermediate metabolizer & chance \\
\hline 6. take tamoxifen & poor metabolizer & $\%$ chance \\
\hline
\end{tabular}

\section{References}

1. Lien EA, Solheim E, Kvinnsland S, et al. Identification of 4-hydroxy-N-desmethyltamoxifen as a metabolite of tamoxifen in human bile. Cancer Res. 1988; 48:2304-2308. [PubMed: 3349495]

2. Desta Z, Ward BA, Soukhova NV, et al. Comprehensive evaluation of tamoxifen sequential biotransformation by the human cytochrome $\mathrm{P} 450$ system in vitro: prominent roles for CYP3A and CYP2D6. J Pharmacol Exp Ther. 2004; 310:1062-1075. [PubMed: 15159443]

3. Goetz MP, Rae JM, Suman VJ, et al. Pharmacogenetics of tamoxifen biotransformation is associated with clinical outcomes of efficacy and hot flashes. J Clin Oncol. 2005; 23:9312-9318. [PubMed: 16361630]

4. Hertz DL, McLeod HL, Irvin WJ. Tamoxifen and CYP2D6: A Contradiction of Data. Oncologist. 2012; 17:620-630. [PubMed: 22531359]

5. Peppercorn J, Hamilton E, Qiu S, et al. Practice and attitudes among US oncologists towards CYP2D6 testing for patients on tamoxifen. Cancer Res. 2009; 69(24 Suppl) Abstract nr 1077.

6. Freund CL, Wilfond BS. Emerging ethical issues in pharmacogenomics: from research to clinical practice. Am J Pharmacogenomics. 2002; 2:273-281. [PubMed: 12421098]

7. Irvin WJ, Carey L, Olajide O, et al. Patients' understanding of a CYP2D6 tamoxifen genotyping study. Cancer Res. 2009; 69(24 Suppl) Abstract nr 6082.

8. Meropol NJ, Weinfurt KP, Burnett CB, et al. Perceptions of patients and physicians regarding phase I cancer clinical trials: implications for physician-patient communication. J Clin Oncol. 2003; 21:2589-2596. [PubMed: 12829680]

9. Agulnik M, Oza AM, Pond GR, et al. Impact and perceptions of mandatory tumor biopsies for correlative studies in clinical trials of novel anticancer agents. J Clin Oncol. 2006; 24:4801-4807. [PubMed: 17050865] 
10. Gurmankin AD, Domchek S, Stopfer J, et al. Patients' resistance to risk information in genetic counseling for BRCA1/2. Arch Intern Med. 2005; 165:523-529. [PubMed: 15767527]

11. Tzeng JP, Mayer D, Richman AR, et al. Women's experiences with genomic testing for breast cancer recurrence risk. Cancer. 2010; 116:1992-2000. [PubMed: 20213682]

12. DeFrank JT, Carey LA, Brewer NT. Understanding how breast cancer patients use risk information from genomic tests. J Behav Med. In press.

13. Irvin WJ, Walko CM, Weck KE, et al. Genotype-guided tamoxifen dosing increases active metabolite exposure in women with reduced CYP2D6 metabolism: a multicenter study. J Clin Oncol. 2011; 29:3232-3239. [PubMed: 21768473]

14. Brewer NT, Edwards AS, O'Neill SC, Tzeng JP, Carey LA, Rimer BK. When genomic and standard test results diverge: Implications for breast cancer patients' preference for chemotherapy. Breast Cancer Research and Treatment. 2009; 117:25-29. [PubMed: 18785002]

15. Lerman C, Hughes C, Croyle RT, et al. Prophylactic surgery decisions and surveillance practices one year following BRCA1/2 testing. Preventive Medicine. 2000; 31:75-80. [PubMed: 10896846]

16. O'Neill SC, Brewer NT, Lillie SE, et al. Women's interest in gene expression analysis for breast cancer recurrence risk. J Clin Oncol. 2007; 25:4628-4634. [PubMed: 17925559]

17. Lidz CW, Appelbaum PS, Grisso T, Renaud M. Therapeutic misconception and the appreciation of risks in clinical trials. Social science and medicine 1982. 2004; 58(9)

18. Sulmasy DP, Astrow AB, He MK, et al. The culture of faith and hope: patients' justifications for their high estimations of expected therapeutic benefit when enrolling in early phase oncology trials. Cancer. 2010; 116:3702-3711. [PubMed: 20564120]

19. Weinfurt KP, Seils DM, Lin L, et al. Research Participants' High Expectations of Benefit in EarlyPhase Oncology Trials: Are We Asking the Right Question? J Clin Oncol. 2012; 30:4396-4400. [PubMed: 23091107]

20. Richman AR, Tzeng JP, Carey LA, et al. Knowledge of genomic testing among early-stage breast cancer patients. Psycho-Oncology. 2011; 20:28-35. [PubMed: 20200857]

21. Partridge AH, Wolff A, Marcom P, et al. The impact of sharing results of a randomized breast cancer clinical trial with study participants. Breast Cancer Res Treat. 2009; 115:123-129.

[PubMed: 18543100] 


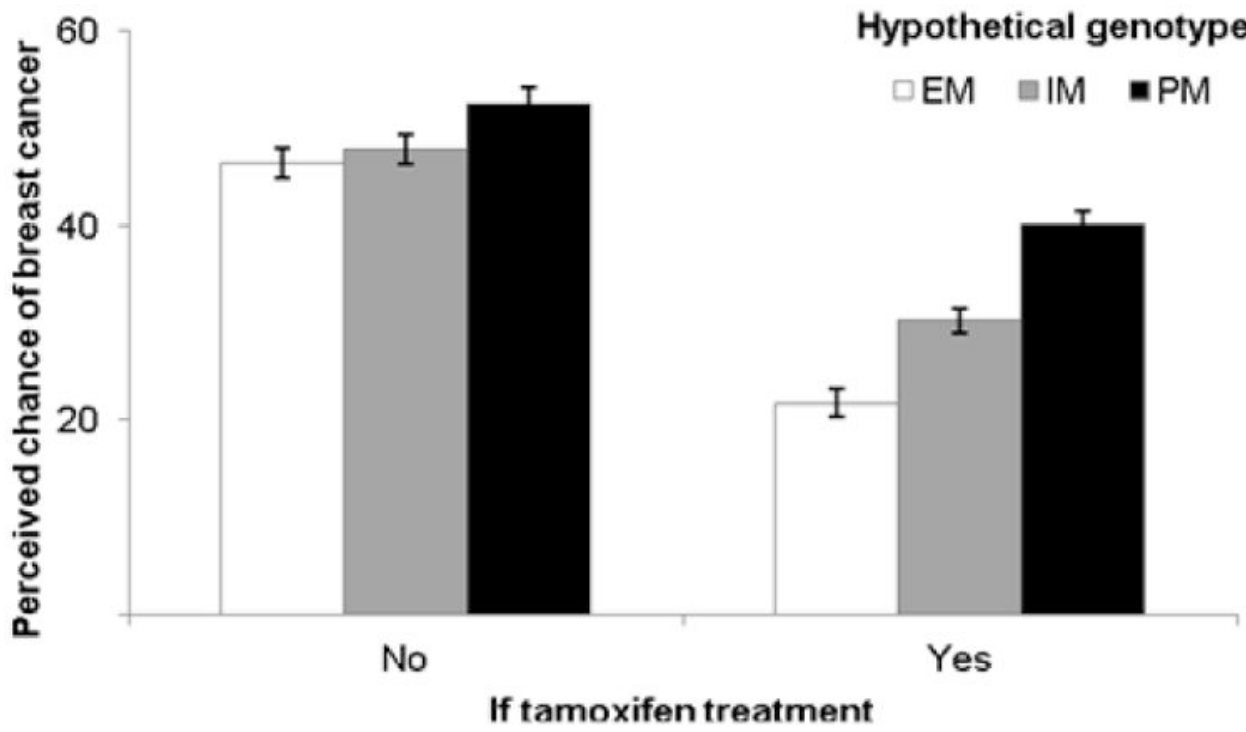

Figure 1.

Effect of hypothetical genotype and tamoxifen treatment on perceived chance of breast cancer recurrence. Error bars report standard errors. 


\section{Table 1}

\section{Participant characteristics}

\begin{tabular}{lcr}
\hline & $\begin{array}{c}\text { Returned incomplete } \\
\text { survey } \\
(\boldsymbol{n}=\mathbf{5 7})\end{array}$ & $\begin{array}{r}\text { Completed survey } \\
(\boldsymbol{n}=\mathbf{3 2 0})\end{array}$ \\
Race & $42(74 \%)$ & $259(81 \%)$ \\
White & $15(26 \%)$ & $61(19 \%)$ \\
Other & $52(46-60)$ & $52(46-61)$ \\
Age in years, median (Q1-Q3) & $21(37 \%)$ & $101(32 \%)$ \\
Premenopausal & $44(77 \%)$ & $272(85 \%)$ \\
Reason for tamoxifen & $10(18 \%)$ & $45(14 \%)$ \\
Treatment of invasive breast cancer & & $111(35 \%)$ \\
Prevention of recurrence (DCIS) & $15(26 \%)$ & $184(58 \%)$ \\
Actual genotype & $37(65 \%)$ & $16(5 \%)$ \\
EM & $3(5 \%)$ & $9(3 \%)$ \\
IM & $2(4 \%)$ & \\
PM &
\end{tabular}

Note. Patients returning complete and incomplete surveys had comparable demographic characteristics (all $p<.05$ ). 